\title{
28 Research Square \\ Identification of Elongation Factor-2 as a Novel Regulator of Mitochondrial Fission
}

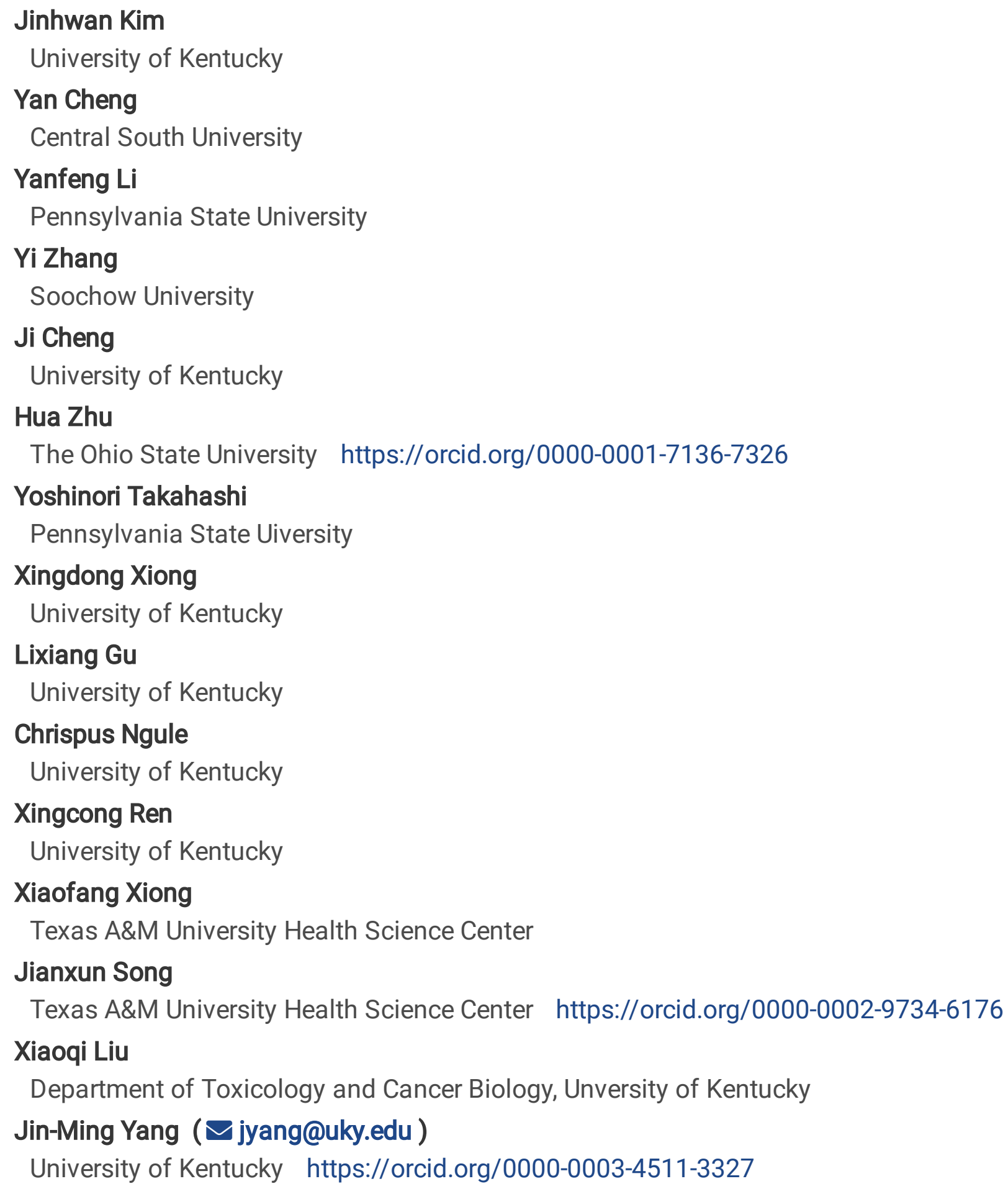

The Ohio State University https://orcid.org/0000-0001-7136-7326

Yoshinori Takahashi

Pennsylvania State Uiversity

\section{Xingdong Xiong}

University of Kentucky

\section{Lixiang Gu}

University of Kentucky

Chrispus Ngule

University of Kentucky

\section{Xingcong Ren}

University of Kentucky

\section{Xiaofang Xiong}

Texas A\&M University Health Science Center

\section{Jianxun Song}

Texas A\&M University Health Science Center https://orcid.org/0000-0002-9734-6176

Xiaoqi Liu

Department of Toxicology and Cancer Biology, Unversity of Kentucky Jin-Ming Yang ( $\square$ jyang@uky.edu )

University of Kentucky https://orcid.org/0000-0003-4511-3327 
Keywords: eEF2, Drp1, mitochondria, dynamics, fission, fusion

Posted Date: August 31st, 2021

DOI: https://doi.org/10.21203/rs.3.rs-778562/v1

License: (c) (1) This work is licensed under a Creative Commons Attribution 4.0 International License. Read Full License 


\section{Abstract}

Mitochondria continuously undergo morphologically dynamic processes of fusion and fission to maintain their size, shape, amount, and function; yet the precise molecular mechanisms by which mitochondrial dynamics is regulated remain to be fully elucidated. Here, we report a previous unappreciated but critical role of eukaryotic elongation factor 2 (eEF2) in regulating mitochondrial fission. eEF2, a G-protein superfamily member encoded by EEF2 gene in human, has long been appreciated as a promoter of the GTP-dependent translocation of the ribosome during protein synthesis. We found unexpectedly in several types of cells that eEF2 was not only present in the cytosol but also in the mitochondria. Furthermore, we showed that mitochondrial length was significantly increased when the cells were subjected to silencing of eEF2 expression, suggesting a promotive role for eEF2 in the mitochondrial fission. Inversely, overexpression of eEF2 decreased mitochondrial length, suggesting an increase of mitochondrial fission. Inhibition of mitochondrial fission caused by eEF2 depletion was accompanied by alterations of cellular metabolism, as evidenced by a reduction of oxygen consumption and an increase of oxidative stress in the mitochondria. We further demonstrated that eEF2 and Drp1, a key driver of mitochondrial fission, co-localized at the mitochondria, as evidenced by microscopic observation, co-immunoprecipitation, and GST pulldown assay. Deletion of the GTP binding motif of eEF2 decreased its association with Drp1 and abrogated its effect on mitochondria fission. Moreover, we showed that wild-type eEF2 stimulated GTPase activity of Drp1, whereas deletion of the GTP binding site of eEF2 diminished its stimulatory effect on GTPase activity. This work not only reveals a previously unrecognized function of eEF2 (i.e., promoting mitochondrial fission), but also uncovers the interaction of eEF2 with Drp1 as a novel regulatory mechanism of the mitochondrial dynamics. Therefore, eEF2 warrants further exploration for its potential as a therapeutic target for the mitochondria-related diseases.

\section{Introduction}

Most eukaryotic cells contain abundant mitochondria, the double membrane-bound, dynamic and complex organelles that take up approximately $25 \%$ of the cytoplasmic volume, and are the key sites for cellular energy production. ${ }^{1}$ In addition to its essential role in generating energy, mitochondria are malleable structures intimately involved in an assortment of cellular processes including cellular redox regulation, cell signaling, calcium homeostasis, cell cycle, cell survival and death; thus, the functions of mitochondria are vital to health and lifespan. ${ }^{2-5}$ Importantly, to maintain their size, shape, number and function, mitochondria continuously undergo morphologically dynamic processes of fusion and fission. ${ }^{6}$ While fusion helps mitigate stress by mixing the contents of partially damaged mitochondria as a way of complementation, fission generates new mitochondria and contributes to quality control by enabling the removal of damaged mitochondria. Under high cellular stress, fission can also promote apoptosis. ${ }^{7,8}$ Therefore, mitochondrial dynamics is critical in controlling the quality of this vital organelle and in ensuring proper inheritance and distribution of mitochondria. Both fission and fusion are highly regulated through their respective machinery. 
Eukaryotic elongation factor-2 (eEF2), encoded by EEF2 gene that localizes on chromosome 19 in humans and a monomeric protein of 858 amino acid residues, is a key regulator of protein synthesis. ${ }^{9}$ eEF2 belongs to the $\mathrm{G}$ protein family, and undergoes conformational changes upon binding to a guanosine nucleotide. ${ }^{10}$ In the translocation process of peptide-tRNA from the ribosomal A-site to P-site, the eEF2-complexed GTP is hydrolyzed and then eEF2 leaves the ribosome as inactive eEF2-GDP. ${ }^{10}$ eEF2 catalyzes the GTP-dependent ribosomal translocation during elongation and during this step, the ribosome changes from the pre-translocational to the post-translocational state as the newly formed Asite-bound peptidyl-tRNA and P-site-bound deacylated tRNA move to the $\mathrm{P}$ and $\mathrm{E}$ sites, respectively. ${ }^{11}$ The activity of eEF2 is regulated by eEF2 kinase, an enzyme phosphorylates eEF2 on Thr-56, and phosphorylation of eEF2 reduces its binding affinity to the ribosome, thus inhibiting mRNA translation. Here, we report a previously unrecognized but important function of eEF2 in regulating mitochondrial fission. We demonstrated that eEF2 is present in the mitochondria as well as in the cytoplasm and supports mitochondrial fission through binding to dynamin-related protein 1 (Drp1) and activating its GTPase activity. The current study not only reveals a novel biological function of eEF2, but also provides a new regulatory pathway of the mitochondrial dynamics.

\section{Results}

\section{eEF2 is present in the mitochondria}

In study of the roles of eEF-2 kinase/eEF2 in stress response, we observed unexpectedly in several types of cells that, while eEF2 kinase was solely detected in the cytosol, eEF2 was found in both cytosol and mitochondria, as determined by Western blot of the mitochondria and cytosol preparations (Fig. 1a). To validate this surprising finding, we performed the mitochondria immunofluorescence staining of eEF2, and showed that eEF2 co-localized with MitoTracker, a fluorescent dye that labels mitochondria within live cells (Fig. 1b). These results provide the clear evidence for the presence of eEF2 in the mitochondria.

\section{Effects of eEF2 expression on the mitochondrial length.}

To determine the functional significance of the presence of eEF2 in the mitochondria, we knocked down eEF2 using RNA interference or overexpressed eEF2 using its expression vector, and then examined whether there were any alterations in morphology of the mitochondria. We observed that as compared with the control cells, MCF-7 cells (Fig. 2a) and mouse embryonic fibroblasts (Fig. 2b) with knockdown of eEF2 had significantly increased mitochondrial lengths, suggesting an inhibition of mitochondrial fission. Similar effect of depletion of eEF2 on mitochondrial length was observed in LN-229 cells (SFig.1). Three eEF2-targeted siRNA were tested, and similar results were obtained (data not shown). Additionally, the electron microscopy (Fig. 2c) and live cell imaging (SFig. 2) showed that the mitochondrial shape was enlarged in the cells with depletion of eEF2, as compared with the control cells. Effect of eEF2 knockdown on mitochondrial fission was also observed under hypoxic condition: while hypoxia promoted mitochondrial fission, knockdown of eEF2 inhibited the fission of this organelle (SFig. 3). By contrast, the mitochondrial length was shortened in the cells subjected to forced expression of eEF2 (Fig. 2d). 
Additionally, the cells with depletion of eEF2 kinase, an enzyme that phosphorylates eEF2, not only showed loss of eEF2 phosphorylation (SFig. 4a) but also had shorter lengths of the mitochondria than the control cells (SFig. 4b), suggesting that the non-phosphorylated form of eEF2 possesses a stronger capacity to support mitochondrial fission. Together, these experiments demonstrate a promotive role of eEF2 in mitochondrial fission.

\section{Effect of eEF2 on mitochondria metabolism}

As mitochondrial dynamics is closely associated with metabolism of this organelle, ${ }^{12}$ we next determined whether the expression of eEF2 affects the mitochondrial metabolism in MCF-7 cells. Fig. 3a shows that as compared with the control cells transfected with a non-targeting siRNA, the cells with knockdown of eEF2 showed a reduction in oxygen consumption rate (OCR), indicating an impaired metabolic activity of the mitochondria. Moreover, the expression of superoxide dismutase 2 (SOD2), an enzyme critical in controlling the mitochondrial reactive oxygen species (ROS), was increased in the cells with knockdown of eEF2 (Fig. 3b), suggesting an increase of oxidative stress that is associated with the impaired mitochondrial function. These results suggest that the coincident changes of OCR and SOD2 in the cells with depletion of eEF2 may be a consequence of altered mitochondrial dynamics.

\section{Interaction of eEF2 with Drp1}

To explore the mechanism by which eEF2 promotes mitochondrial fission, we first examined the effect of eEF2 expression on Drp1, a dynamin-related GTPase that acts as a key driver of mitochondrial fission. We found that the amount of mitochondrial Drp1 protein was remarkably down-regulated, whereas the total or cytosol Drp1 protein was unchanged, in the cells subjected to knockdown of eEF2 expression (Fig. 4a). By contrast, the mitochondrial Drp1 protein was increased in the cells transfected with an eEF2 expression plasmid (Fig. 4b). These results suggest that eEF2 is crucial in supporting Drp1 translocation to the mitochondria. Next, we tested the possible association of eEF2 with Drp1 using the approaches of co-immunoprecipitation and GST-associated precipitation. Co-immunoprecipitation experiments demonstrated that eEF2 and Drp1 are physically associated (Fig. 4c), and GST-associated precipitation using eEF2 or Drp1 GST fusions proteins also showed the binding of eEF2 to Drp1 (Fig. 4d). Moreover, we observed that eEF2 and Drp1 co-localized at the mitochondria, as analyzed by immunofluorescence staining and confocal microscopy (Fig. 4e). These results imply a physical association of eEF2 with Drp1 and suggest that the effect of eEF2 on mitochondrial fission may be mediated through its interaction with Drp1. As GTPase activity of Drp1 is essential for mitochondrial fission and eEF2 has GTP binding site, we asked whether eEF2 interacts with Drp1 through its GTP binding domain. To test this possibility, we constructed an eEF2 mutant in which the GTP binding motif (104-108; SPGHV) of eEF2 was deleted (Fig. 6a) and transfected this expression vector of the mutant into HEK293 cells. We showed that deletion of the eEF2 GTP binding motif decreased the association of eEF2 with Drp1 in vivo (Fig. 4c) as well as in vitro (Fig. 4d). To further validate the role of eEF2 in promoting mitochondrial fission, we tested the effect of the deletion mutant of eEF2 on mitochondrial length. As shown in Fig. 5a, this deletion mutant had no effect on mitochondria fission as compared with wild-type eEF2. These results suggest that the effect of 
eEF2 on mitochondrial fission is mediated through its interaction with Drp1 at its GTP binding site. To confirm the specificity of the effect of eEF2 on mitochondrial fission, we performed rescue experiments using an RNAi-resistant eEF2 mutant in which 3 synonymous, single nucleotide changes were incorporated into the RNAi target sequence of wild-type eEF2 cDNA. Fig. $5 \mathbf{b}$ shows that the effect of eEF2-targeted siRNA on the mitochondrial length was partially rescued in the cells transfected with this RNAi-resistant mutant of eEF2.

\section{Activation of GTPase of Drp1 by eEF2}

Next, we determined and compared the effects of eEF2 and its deletion mutant (Fig. 6a) on GTPase activity of Drp1, using the purified proteins in the in vitro GTPase assay. Fig. $\mathbf{6 b}$ shows that while wildtype eEF2 could substantially increase Drp1 GTPase activity in a concentration-dependent manner, the eEF2 mutant that lacks GTP binding motif had little stimulating effect on GTPase of Drp1. These results further support the role of eEF2-Drp1 interaction in promoting mitochondrial fission, which is driven by Drp1 through its GTPase activity (Fig. 6c).

\section{Discussion}

Thus far, the only known function of eEF2 is its role in mRNA translation by facilitating the GTPdependent translocation of the ribosome. Unexpectedly, we found that, in addition to its presence in the cytoplasm, eEF2 also exists in the mitochondria (Fig. 1) and interacts with Drp1 to promote mitochondrial fission (Fig. 4). Although these novel findings appear to stray from the current knowledge about eEF2, they clearly show that this protein has an important regulatory role in mitochondrial dynamics. Noteworthily, novel functions of other elongation factors have reported recently. For instance, it was found that elongation factor-Tu could specifically bind to T6S integral membrane toxin, and this interaction is required for delivery of this toxin to target cells. ${ }^{13}$ This finding provides another evidence that elongation factors may have the aptitude for interacting with other proteins, affecting certain cellular functions.

It was shown in a previous study that silencing of eEF2 expression caused morphological alterations of mitochondria and other organelles, ${ }^{14}$ but no further investigation on the role of eEF2 in mitochondria regulation has been reported so far. Here, we show that eEF2 is not only present in the cytoplasm, but also exists in the mitochondria and has a promotive role in mitochondrial fission. The positive regulation of mitochondrial fission by eEF2 was evidenced by our observation that knockdown of eEF2 increased the length of mitochondria (Fig. 2a, b and c) and forced expression of eEF2 decreased the length of this organelle (Fig. 2d). Expression of eEF2 also affected mitochondrial metabolism (Fig. 3) and cell proliferation (SFig. 5), the likely consequences of the altered mitochondrial fission mediated by eEF2 expression. Mechanistically, eEF2 appears to stimulate the activity of GTPase of Drp1 through its interaction with this major component of the fission machinery, as these two proteins are physically associated and co-localized (Fig. 4), and deletion of the GTP binding site of eEF2 substantially weakened 
its binding to Drp1 (Fig. 4) and impaired its ability to activate GTPase of Drp1 (Fig. 6) and to support mitochondrial fission (Fig. 5).

Drp1 is a cytosolic protein, which upon activation translocates to the outer membrane of the mitochondria and plays a central role in inducing mitochondrial fission. Nevertheless, how precisely Drp1 is targeted and translocated from the cytosol to the mitochondria remains less clear. We show here that silencing eEF2 expression not only decreased the mitochondrial eEF2 but also Drp1 in the mitochondria (Fig. 4b); by contrast, forced expression of eEF2 increased the mitochondrial Drp1 (Fig. 4a). Therefore, it is likely that binding of eEF2 to Drp1 facilitates the translocation of Drp1 to the outer membrane of the mitochondria. It is known that phosphorylation of eEF2 by eEF2 kinase reduces its binding affinity to the ribosome, thereby inhibiting peptide elongation. Our experiments showed that the cells with depletion of eEF2 kinase had a significantly increase of mitochondrial fission (SFig. 4b), ${ }^{15}$ suggesting that phosphorylation of eEF2 limits its interaction with Drp1. Additionally, it was reported that eEF2 can be phosphorylated by cyclin A-CDK2 at Se-595; thus, cyclin A-CDK2 might affect the eEF2-mediated mitochondrial fission as well.

As mitochondrial fusion and fission are important determinants of the functions of this organelle and defects in these processes are implicated in various human diseases such as cardiovascular, neurodegenerative, endocrine and neoplastic diseases, ${ }^{16-18}$ our revelation of eEF2 as a new regulator of mitochondrial dynamics might provide a potential target for therapeutic intervention of those human disorders resulting from mitochondria dysfunction. Notably, small molecule inhibitors of eEF2 have just been developed recently, ${ }^{19}$ and this may provide a promising opportunity to test their effects on the mitochondrial activity and the mitochondria-associated biological functions and their potential as therapeutic agents for the mitochondria-associated diseases.

\section{Materials And Methods}

\section{Cell cultures}

Human breast cancer cell lines MCF7 and MDA-MB-231, glioblastoma LN229, human astroglia SVGp12, mouse embryonic fibroblast line MEF, and mouse melanoma cell line B16, were purchased from ATCC and maintained in Dulbeco modified Eagle's medium (DMEM) (Sigma) supplemented with $10 \%$ (v/v) fetal bovine serum, $100 \mathrm{U} / \mathrm{ml}$ penicillin, and $100 \mu \mathrm{g} / \mathrm{ml}$ streptomycin.

\section{RNA interference}

The siRNA specific to the human eEF2 and mouse eEF2, and the scrambled siRNA were prepared by Dharmacon. The following specific double strands were used: human, 5'GGUGUUUGAUGCGAUCAUGAAUUTC-3' (sense), 5'-GAAAUUCAUGAUCGC AUCAAACACCUU-3' (anti-sense); mouse, 5'-GGUGU UUGAUGCGAUCAUGAAUUTC-3' (sense), 5'-GAAAUUCA UGAUCGCAUCAAACACC UU-3' (antisense). siRNAs were introduced into cells using RNAi MAX (Invitrogen) according to the manufacturer's protocol. 


\section{Construction of plasmids}

To construct eEF2 plasmids, the cDNAs were synthesized from total RNA (4 $\mu$ g) of MCF10A cells, using the Super Script IV reverse transcriptase (Life Technologies). The human eEF2 gene was delivered to pcDNA3.1 His6C vector, using BamHI and Xhol restriction enzyme site. The domains of the GTP binding motifs were analyzed using Uniport analyzer (https://www.uniprot.org/uniprot/P13639). The following primers were used: human eEF2, forward: 5'-GACGGATCCGCCACCATGGTGAACTTCACGGTAG-3'; reverse: 5'- ACCTCGA GCGGCTACAATTTGTCCAGGAAGTTG-3'. eEF2 GTP binding motif mutant (CATCAACCT CATTGACTCCCCCGGGCATGTCGACTTCTCCT): forward: 5'-CATCAACCTCATTGTCGAC TTCTCCTCG-3'; reverse: 5'-CGAGGAGAAGTCGACAATGAGGTTGATG-3'. To produce eEF2-GST fusion protein, the vectors of eEF2 and eEF2 GTP binding mutant were digested by BamHI and Xhol and subcloned into pGEX-4T-2 vector. To produce Drp1-GST fusion protein, the human Drp1 gene from pGW1-Drp ${ }^{20}$ was amplified by PCR using the following primers; forward: 5'-TTTGGATCCATGGAGGCGCTAATTCC-3' and reverse: 5'TTTCTCGAGTCACC AAAGATGAGTCTCCC-3' and then cloned into pGEX-4T-2 vector.

The plasmid of eEF2 RNAi-resistant mutant was prepared using two-step PCR techniques as previously reported. ${ }^{21}$ Briefly, the construct was amplified using the following primers: 5'-

AAGGTGTTTGATGCGATCATGAACTTIAAAAAAGAGGA-3' (forward) and 5'- TCCTCTTTT ITAAAGITCATGATCGCATCAAACACCTT-3' (reverse). Three nucleotides in the target sequence of siRNA were changed, and the resultant human eEF2 CDNAs was then subcloned into pcDNA3.1-His $6 \mathrm{C}$ vector. In this RNAi-resistant mutant, the target sequence, GAAITTCAAGAAA, was mutated with 3 synonymous, single nucleotide polymorphisms to the following sequence: GAAAAㄷTTIAAAAAA.

\section{Isolation of mitochondria}

Cells were suspended in mitochondria isolation buffer $(250 \mathrm{mM}$ sucrose, $0.1 \mathrm{mM}$ EDTA, and $2 \mathrm{mM}$ HEPES, pH 7.4) containing protease inhibitors and phosphatase inhibitors (Thermo Scientific) and homogenized using $21 \mathrm{G}$ needle. The homogenized cells were then centrifuged at 3,000 rpm for $10 \mathrm{~min}$ at $4{ }^{\circ} \mathrm{C}$. The mitochondria-containing supernatants were transferred to new tubes and centrifuged at 15,000 $\mathrm{rpm}$ for $30 \mathrm{~min}$ at $4{ }^{\circ} \mathrm{C}$. After centrifugation, the pellets (mitochondrial fraction) were lysed with laemmli sample buffer (Bio-Rad).

\section{Measurement of mitochondria length}

Cells plated ion $12 \mathrm{~mm}$ coverslips were incubated with $0.5 \mu \mathrm{M}$ Mitotracker Red (Invitrogen) at $37^{\circ} \mathrm{C}$ for 30 min, and then fixed with $4 \%$ formaldehyde. The stained cells were mounted on glass slides using CYTOSEAL 60 mounting medium (Thermo Scientific). MitoTracker Red intensity was observed at $581 \mathrm{~nm}$ (excitation) using a laser scanning confocal microscope (Nikon). Quantification of the mitochondria length was performed on least 5 images using NIS-elements AR Analysis software (Nikon).

\section{Western blot}


Cell lysates were sonicated using Virsonic 100 (1-3 watts) for 5 seconds, and then centrifuged at 15000 rpm for 20 min at $4{ }^{\circ} \mathrm{C}$. Protein amounts were measured using a BCA protein assay kit (Pierce). The proteins $(25 \mu \mathrm{g})$ were separated by SDS-PAGE, and then transferred onto PVDF membrane. The membranes were incubated with primary antibodies for overnight in $5 \%$ BSA at $4{ }^{\circ} \mathrm{C}$, followed by incubation with a secondary antibody for $2 \mathrm{hrs}$ at room temperature. A femto-chemiluminescence kit (Thermo Fisher Scientific) was used for visualization of the signal. Western blots were analyzed using Image Lab software (Bio-Rad). The antibodies for eEF2 (rabbit), phospho-eEF2 (T65) (rabbit), eEF2K (rabbit), Drp1 (rabbit), SOD2 (rabbit), HA tag (mouse), and prohibitin 1 (PHB1, rabbit) were purchased from Cell signaling. GAPDH antibody (mouse) was purchased from Santa Cruz biotechnologies. $6 \mathrm{X}$ His antibody (mouse) was purchased from Clontech.

\section{Immunoprecipitation}

HEK293 cells were transfected with pcDNA, pcDNA eEF2, pcDNA eEF2 GTP mutant, and pGW1-Drp1 using lipofectamin 3000 reagent (Invitrogen) for 3 days according to the manufacturer's protocol. The eEF2 and Drp1 antibodies were conjugated with protein A/G agarose bead for overnight at $4{ }^{\circ} \mathrm{C}$. Anti-lgG (Rabbit) used as a control (Santa Cruz biotechnologies). The beads were washed three time in ice cold $1 \mathrm{X}$ TBST and incubated with $500 \mu \mathrm{g}$ whole cell extracts of pcDNA, eEF2, eEF2 GTP mutant, and Drp1 for $24 \mathrm{hrs}$ at $4{ }^{\circ} \mathrm{C}$. After washing by $1 \mathrm{X}$ TBST three times, antibody-conjugated proteins were retained from the beads using SDS-sample buffer (Bio-Rad) and were analyzed by western blot.

\section{GST pull-down assay}

The plasmids of human GST-eEF2, GST-eEF2 GTP mutant and GST-Drp1 were constructed as described above, and the fusion proteins of GST-eEF2, GST-eEF2 GTP mutant and GST-Drp1 were prepared as previously described. ${ }^{22}$ The fusion proteins $(500 \mu \mathrm{g})$ were incubated with GST-magnetic beads (50 $\left.\mu \mathrm{l}\right)$ for 24 hours at $4{ }^{\circ} \mathrm{C}$. After washing with $1 \mathrm{X}$ PBST, the GST fusion protein-conjugated beads were incubated with the extracts of HEK 293 cells transfected with eEF2 or eEF2 GTP mutant, and Drp1, for another 24 hours. The proteins retained on the beads were examined by western blot.

\section{Immunofluorescence microscopy}

Cells plated on cover slides were first incubated with $0.5 \mu \mathrm{M}$ Mitotracker Red (Invitrogen) at $37^{\circ} \mathrm{C}$ for 30 min, and then fixed with $4 \%$ formaldehyde. Following treatment with blocking buffer, the cells were incubated with an eEF2 antibody at $4{ }^{\circ} \mathrm{C}$ for overnight, followed by incubation with the second antibodies, AX568 Fluor Red and Alexa Fluor 488 Green, at room temperature for 90 min. Finally, the sample slides were mounted with ProLong Gold Antifade Reagent (Life Technologies) with or without DAPI and incubated overnight at room temperature. The slides were observed using a laser scanning confocal microscope (Nikon).

\section{Electron microscopy}


Cells were harvested by trypsinization, and then fixed and embedded in spur resin. Ninety $\mathrm{nm}$ - thin sections of cell pellets were cut and examined at $80 \mathrm{Kv}$ with a JEOL 1200 EX transmission electron microscope.

\section{Oxygen consumption rate (OCR)}

Oxygen consumption rate (OCR) was measured using the XFe96 analyzer (Agilent Technologies). Briefly, cells ( $4 \times 10^{4}$ cells) were plated into wells of an XFe96 cell culture microplate and incubated at 37 खC in a $\mathrm{CO}_{2}$ incubator for 24 hours. The assay was started after cells were equilibrated for 1 hour in XF assay medium supplemented with $10 \mathrm{mM}$ glucose, $1 \mathrm{mM}$ sodium pyruvate and $2 \mathrm{mM}$ glutamine. OCR activity was monitored through sequential injections of $1 \mu \mathrm{M}$ oligomycin, $0.6 \mu \mathrm{M} \mathrm{FCCP}$, and $1 \mu \mathrm{M}$ rotenone/ antimycin A to calculate basal, coupled, maximal respiration and spare respiratory capacity. Each plotted value was normalized either to cell counts. Agilent Seahorse XF Analyzer measures oxygen consumption rate (OCR) at intervals of approximately 5-8 min. OCR data were evaluated using 8 individual well values.

\section{Drp1 GTPase assay}

The plasmids of pGEX-4T-2/eEF2, pGEX-4T-2/eEF2 GTP binding mutant and pGEX-4T-2/Drp1 were conditionally expressed in Stbl3 E. coli cells with $200 \mathrm{mM}$ of isopropylthio- $\beta$-galactoside (IPTG) for 4 hours. Then, the cells were centrifuged at 5,000 rpm for $30 \mathrm{~min}$, and the pelleted cells were suspended in nucleotide and phosphate free solution (20 mM Tris- $\mathrm{Cl} \mathrm{pH} 7.5$ and $0.01 \%$ Triton X-100) and sonicated. After sonication, the cell lysates were centrifuged at $15,000 \mathrm{rpm}$ for $30 \mathrm{~min}$. The cell extracts were incubated with $50 \mu \mathrm{l}$ of GST-magnetic bead for 24 hours on an orbital shaker at $4{ }^{\circ} \mathrm{C}$. The GST-fusion proteins were eluted using $50 \mu$ l of elution buffer $(50 \mathrm{mM}$ Tris- $\mathrm{Cl}(\mathrm{pH} 8.8), 15 \mathrm{mM}$ glutathione and $1 \mathrm{mM}$ EDTA). The purified proteins, Drp1 and eEF2 or Drp1 and eEF2 GTP deletion mutant, were mixed (vol:vol: $1: 1=2 \mu \mathrm{g}: 2 \mu \mathrm{g}$ ) for GTPase assay. The GTPase activity of Drp1 was measured using the colorimetric GTPase activity assay kit (Sigma) following the manufacture's protocol.

\section{Statistical analysis}

All experiments were performed in triplicates and each experiment was repeated at least three times. The data shown were the mean \pm standard deviation (S.D.) of a representative experiment. Student's $t$ - test was used to analyze the difference between two means. The difference was considered significant when the $p$ values: ${ }^{*}, p<0.05 ;{ }^{* *}, p<0.01 ;{ }^{\star \star \star}, p<0.001$.

\section{Declarations}

\section{Acknowledgements}

We thank Dr. Peng-peng Zhu (National Institute of Neurological Disorders and Stroke) for providing the pGW1-Drp1 plasmid. This research was supported by the Redox Metabolism Shared Resource Facility of the University of Kentucky Markey Cancer Center (P30CA177558) and by a startup fund from College of 
Medicine and Markey Cancer Center, University of Kentucky. We also thank the Imaging Core Facility of the Center for Cancer Metabolism COBRE (P20 GM121327) for providing the imaging systems.

\section{References}

1. Janikiewicz, J. et al. Mitochondria-associated membranes in aging and senescence: structure, function, and dynamics. Cell Death Dis 9, 332 (2018).

2. Kim, Y. et al. Mitochondria, Metabolism, and Redox Mechanisms in Psychiatric Disorders. Antioxid Redox Signal 31, 275-317 (2019).

3. Picca, A. et al. Mitochondria and redox balance in coeliac disease: A case-control study. Eur J Clin Invest 48 (2018).

4. Fulda, S. Mitochondria, redox signaling and cell death in cancer. Biol Chem 397, 583 (2016).

5. Seo, A.Y. et al. New insights into the role of mitochondria in aging: mitochondrial dynamics and more. J Cell Sci 123, 2533-2542 (2010).

6. Arakaki, N. et al. Dynamics of mitochondria during the cell cycle. Biol Pharm Bull 29, 1962-1965 (2006).

7. Tatsuta, T. \& Langer, T. Quality control of mitochondria: protection against neurodegeneration and ageing. EMBO J 27, 306-314 (2008).

8. Berman, S.B. \& Hollenbeck, P.J. Exploring the life cycle of mitochondria in neuropsychiatric diseases: mitochondrial dynamics and quality control. Neurobiol Dis 51, 1-2 (2013).

9. Ryazanov, A.G., Shestakova, E.A. \& Natapov, P.G. Phosphorylation of elongation factor 2 by EF-2 kinase affects rate of translation. Nature 334, 170-173 (1988).

10. Davydova, E. et al. Identification and characterization of a novel evolutionarily conserved lysinespecific methyltransferase targeting eukaryotic translation elongation factor 2 (eEF2). J Biol Chem 289, 30499-30510 (2014).

11. Susorov, D. et al. Eukaryotic translation elongation factor 2 (eEF2) catalyzes reverse translocation of the eukaryotic ribosome. J Biol Chem 293, 5220-5229 (2018).

12. Aishwarya, R. et al. Pleiotropic effects of mdivi-1 in altering mitochondrial dynamics, respiration, and autophagy in cardiomyocytes. Redox Biol 36, 101660 (2020).

13. Whitney, J.C. et al. An interbacterial $\operatorname{NAD}(P)(+)$ glycohydrolase toxin requires elongation factor Tu for delivery to target cells. Cell 163, 607-619 (2015).

14. Chen, C.Y. et al. Sumoylation of eukaryotic elongation factor 2 is vital for protein stability and antiapoptotic activity in lung adenocarcinoma cells. Cancer Sci 102, 1582-1589 (2011).

15. Hizli, A.A. et al. Phosphorylation of eukaryotic elongation factor 2 (eEF2) by cyclin A-cyclindependent kinase 2 regulates its inhibition by eEF2 kinase. Mol Cell Biol 33, 596-604 (2013).

16. Chan, D.C. Mitochondrial fusion and fission in mammals. Annu Rev Cell Dev Biol 22, 79-99 (2006). 
17. Hall, A.R., Burke, N., Dongworth, R.K. \& Hausenloy, D.J. Mitochondrial fusion and fission proteins: novel therapeutic targets for combating cardiovascular disease. Br J Pharmacol 171, 1890-1906 (2014).

18. Chen, H. \& Chan, D.C. Mitochondrial dynamics-fusion, fission, movement, and mitophagy-in neurodegenerative diseases. Hum Mol Genet 18, R169-176 (2009).

19. Baragana, B. et al. A novel multiple-stage antimalarial agent that inhibits protein synthesis. Nature $522,315-320$ (2015).

20. Zhu, P.P. et al. Intra- and intermolecular domain interactions of the C-terminal GTPase effector domain of the multimeric dynamin-like GTPase Drp1. J Biol Chem 279, 35967-35974 (2004).

21. Aizawa, M. \& Fukuda, M. Small GTPase Rab2B and Its Specific Binding Protein Golgi-associated Rab2B Interactor-like 4 (GARI-L4) Regulate Golgi Morphology. J Biol Chem 290, 22250-22261 (2015).

22. Bourdoncle, A. et al. The nuclear receptor coactivator PGC-1 alpha exhibits modes of interaction with the estrogen receptor distinct from those of SRC-1. J Mol Biol 347, 921-934 (2005).

\section{Figures}


a

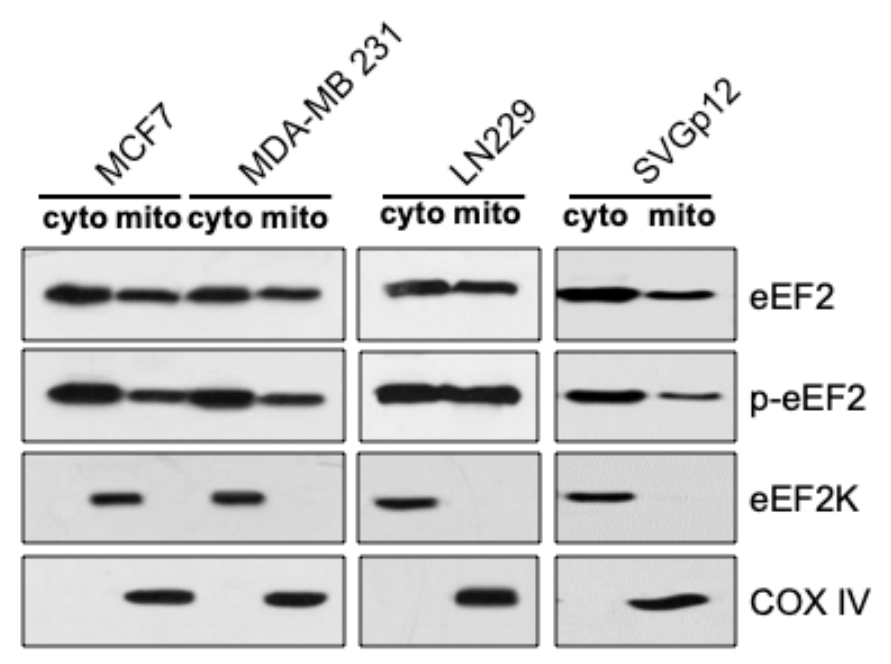

b
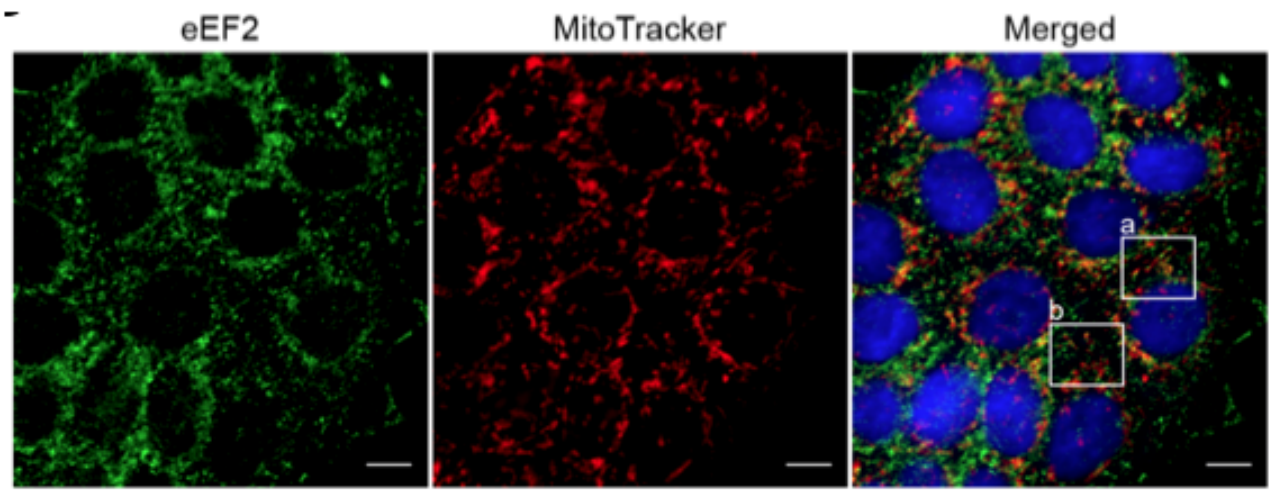

a

b
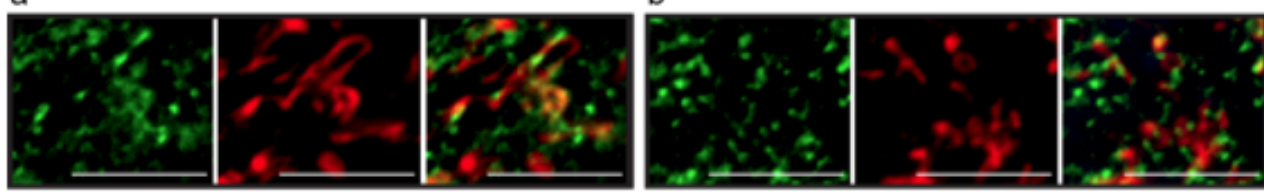

Green: eEF2 Red: MitoTracker

\section{Figure 1}

eEF2 is detected in the mitochondria. a. The mitochondria and cytosolic fractions of MCF7, MDA-MB-231, LN229, or SVGp12 cells were separated and eEF2, p-eEF2 and eEF2 kinase were examined by western blot. COX IV was used as a marker of mitochondria fraction. b. MCF7 cells were incubated with Mitotracker Red® and eEF2 antibody, followed by incubation with a second antibody and AlexaFluorß488 Green. Green: eEF2; red: mitochondria. 
Figure 2

a
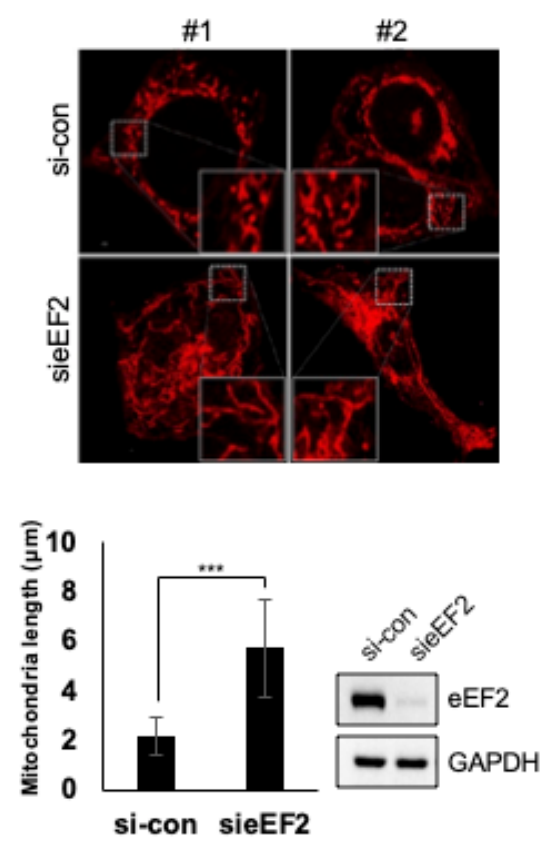

C

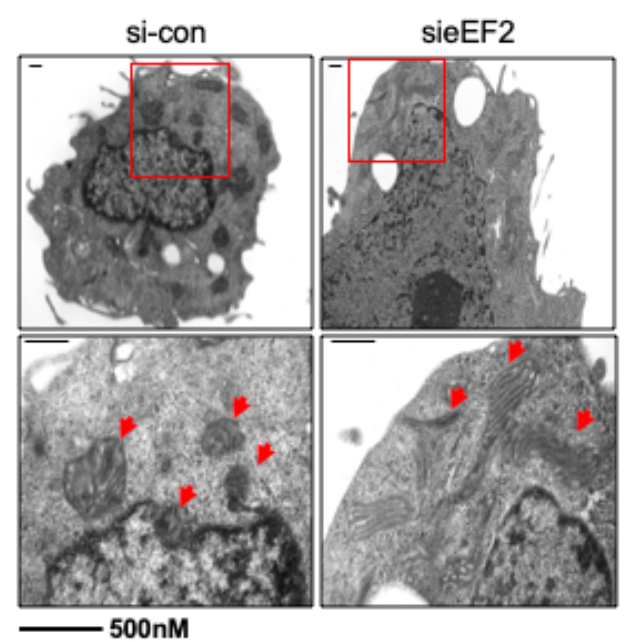

b
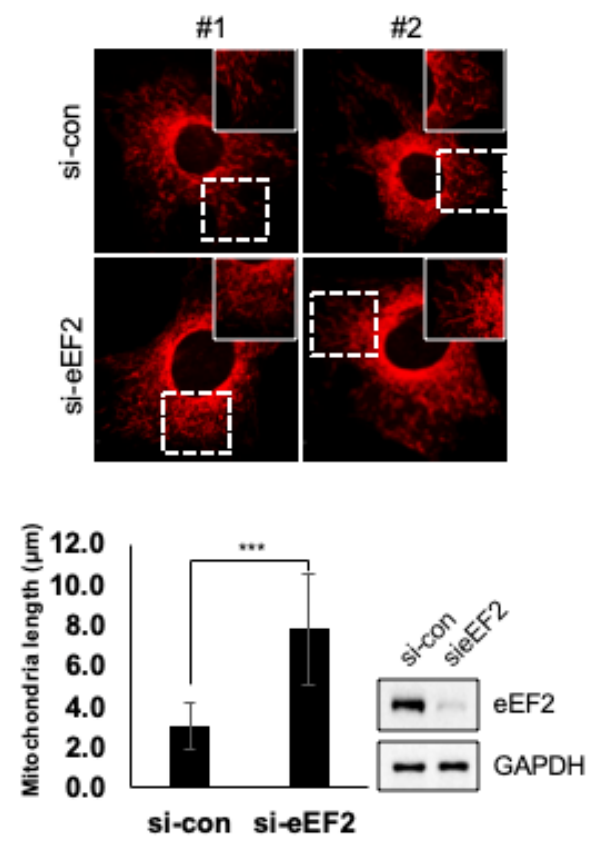

d
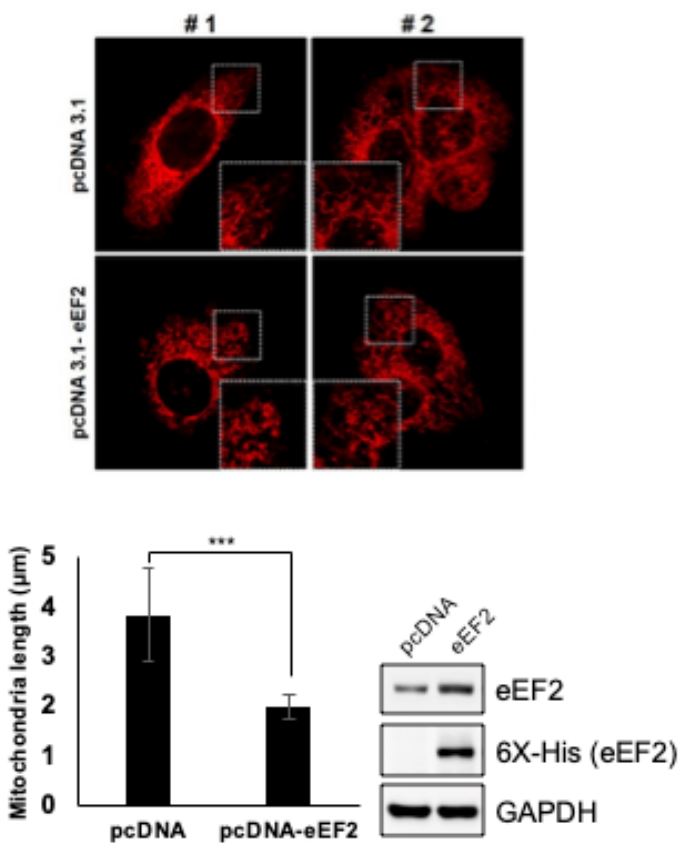

\section{Figure 2}

Effect of eEF2 expression on the length of mitochondria. MCF7 cells (a) or MEFs (b) were transfected with sieEF2 or non-targeting control RNA for 72 hours, and then were stained with Mitotracker Red® and observed under a confocal microscope. The lengths of mitochondria were measured using the NISelements AR Analysis software (Nikon); five independent images were used for measuring the mitochondria length $(n=5)$. c. MCF7 cells with or without silencing of eEF2 expression were harvested by 
trypsinization, then were fixed and embedded in spur resin. Ninety $\mathrm{nm}$ thin sections were cut and examined at $80 \mathrm{Kv}$ under a JEOL $1200 \mathrm{EX}$ transmission electron microscope. The bar: $500 \mathrm{~nm}$ scale. $\mathrm{d}$. MCF7 cells were transfected with pcDNA eEF2 expression vector or control pcDNA 3.1 His6C vector for 72 hours, and then were stained with Mitotracker Red $\circledast$ and observed under a confocal microscope. The lengths of mitochondria were measured using the NIS-elements AR Analysis software (Nikon); five independent images were used for measuring the mitochondria length $(n=5)$.

Figure 3

a

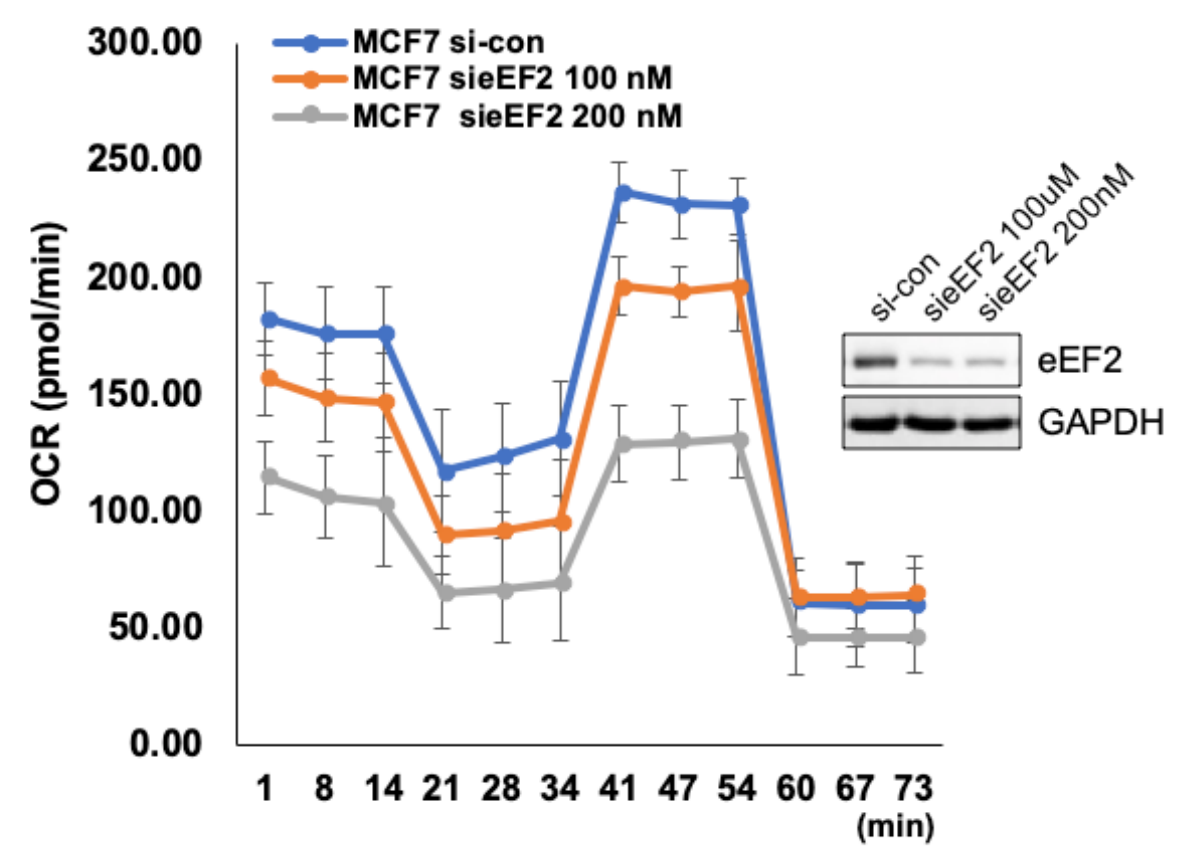

b

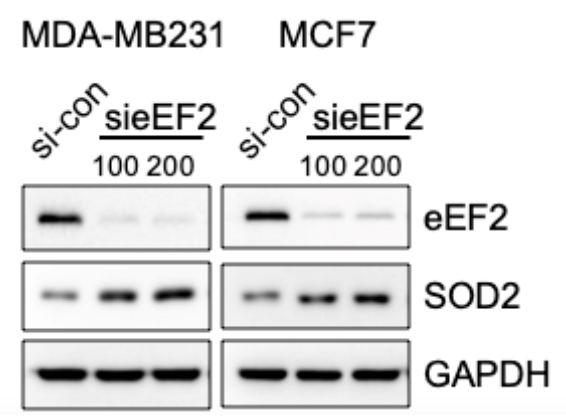

Figure 3 
Knockdown of eEF2 alters mitochondrial metabolism. a. MCF7 cells were transfected with 100 or $200 \mathrm{nM}$ of sieEF2 or non-targeting control RNA for 72 hours. The transfected cells were then seeded in 96-well plates (4 X 104/well). OCR were time-serially analyzed using an XFe96 analyzer. b. MDA-MB-231 or MCF7 cells were transfected with 100 or $200 \mathrm{nM}$ of sieEF2 or non-targeting control RNA for 72 hours, followed by western blot analysis of eEF2 and SOD2. GAPDH is used as a loading control.

Figure 4

a

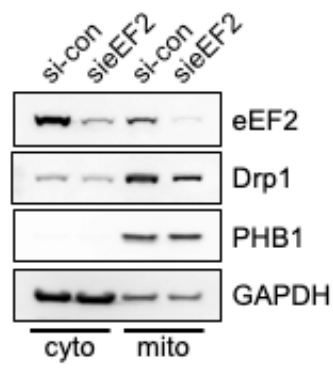

b

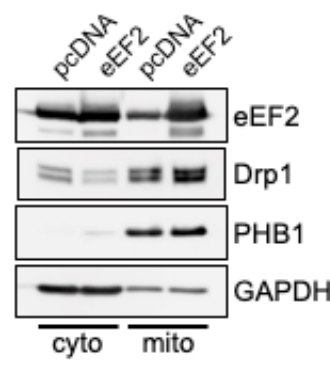

d
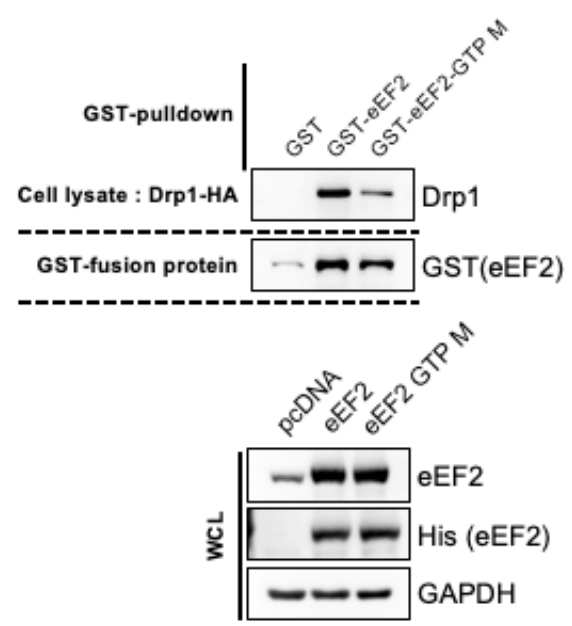

C
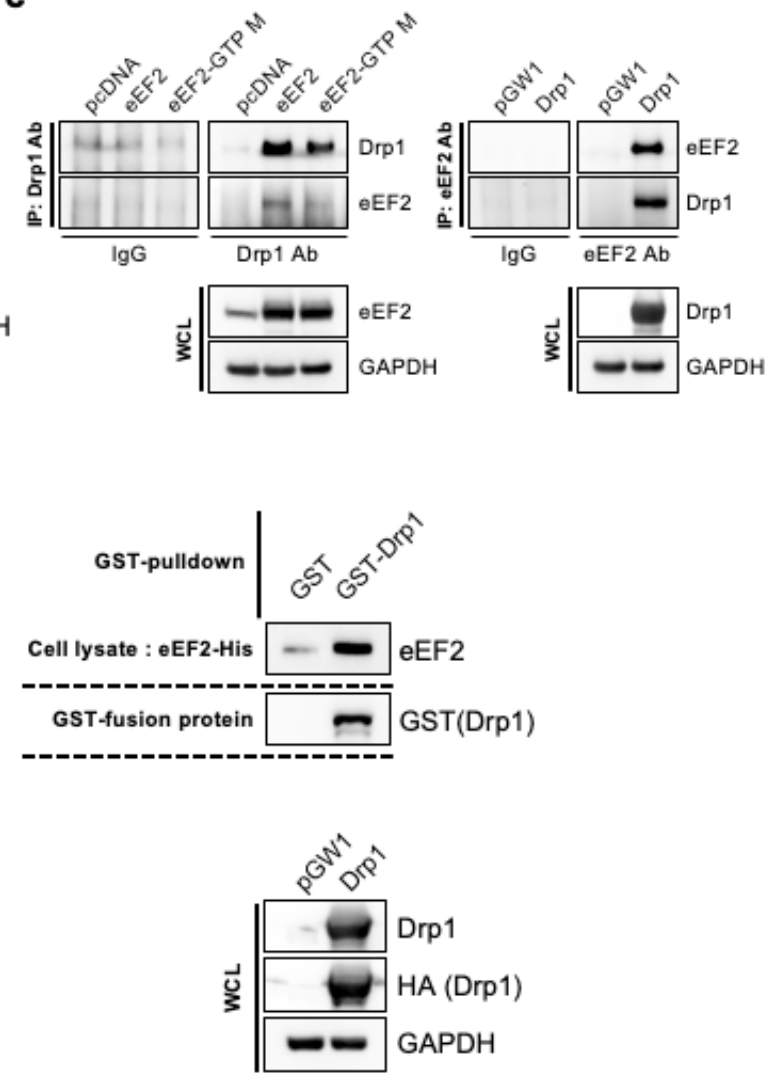

e

Tom20/Drp1/eEF2
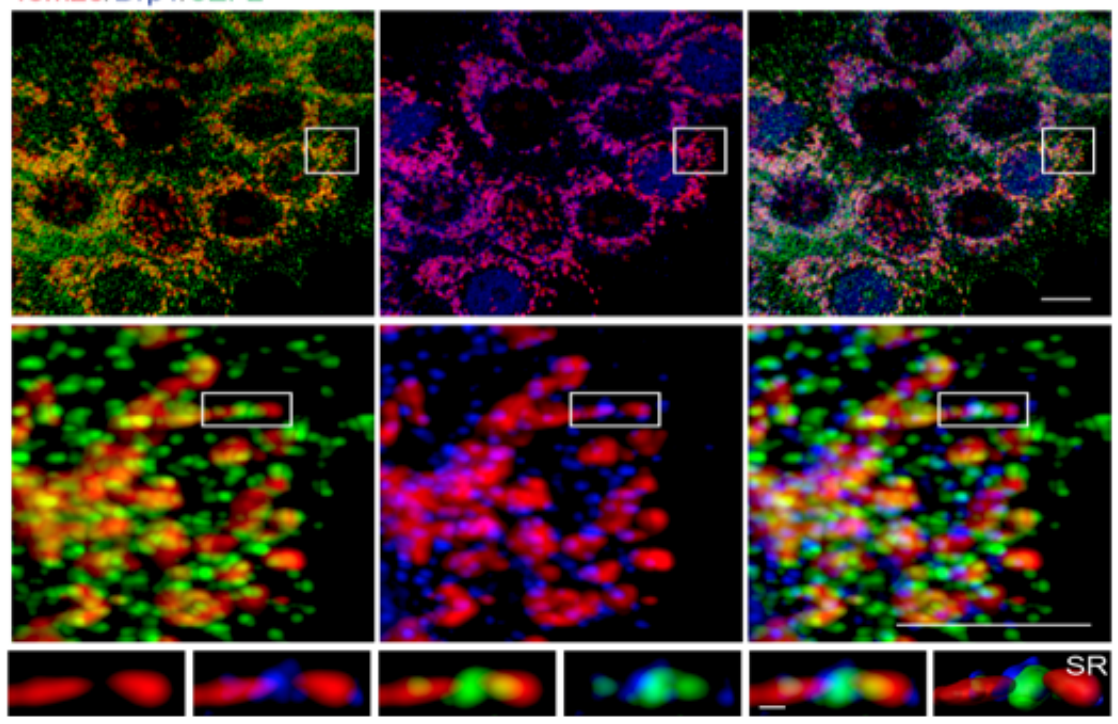

Figure 4 
eEF2 binds to and colocalizes with Drp1 on the mitochondria. a. HEK293 cells were transfected with pcDNA 3.1 His6C or pcDNA eEF2 for 72 hours. The cytosol and mitochondria fractions were prepared for western blot analysis. b. HEK293 cells were transfected with sieEF2 or non-targeting control RNA for 72 hours. The cytosol and mitochondria fractions were prepared for western blot analysis. PHB1 (prohibitin 1) was used as a mitochondrial marker. c. HEK 293 cells were transfected with pcDNA eEF2 or eEF2 GTP mutant (M) and pGW1 containing Drp1 for 72 hours. Left upper panel: The agarose-conjugated Drp1 antibody was incubated with the lysates of the cells transfected with the pcDNA 3.1 His containing eEF2 or eEF2 GTP mutant for 24 hours. The eluted protein complex was examined by western blot. Right upper panel: The agarose-conjugated eEF2 antibody was incubated with the lysates of the cells overexpressing Drp1 for 24 hours. The eluted protein complex was examined by Western blot. Rabbit IgG was used as a control. d. GST-eEF2, GST- eEF2 GTP mutant, and GST-Drp1 proteins were precipitated with magnetic GST beads, and then incubated with the lysates of the cells transfected with either eEF2, eEF2 GTP mutant or Drp1. The eluted proteins were analyzed by Western blot. e. MCF7 cells were seeded in coverslip and incubated with eEF2 and Drp1 antibodies, followed by incubation with the second antibodies, Alexa Fluor ${ }^{\circledR} 488$ Green and AX568 Fluor®Red. 
Figure 5

a
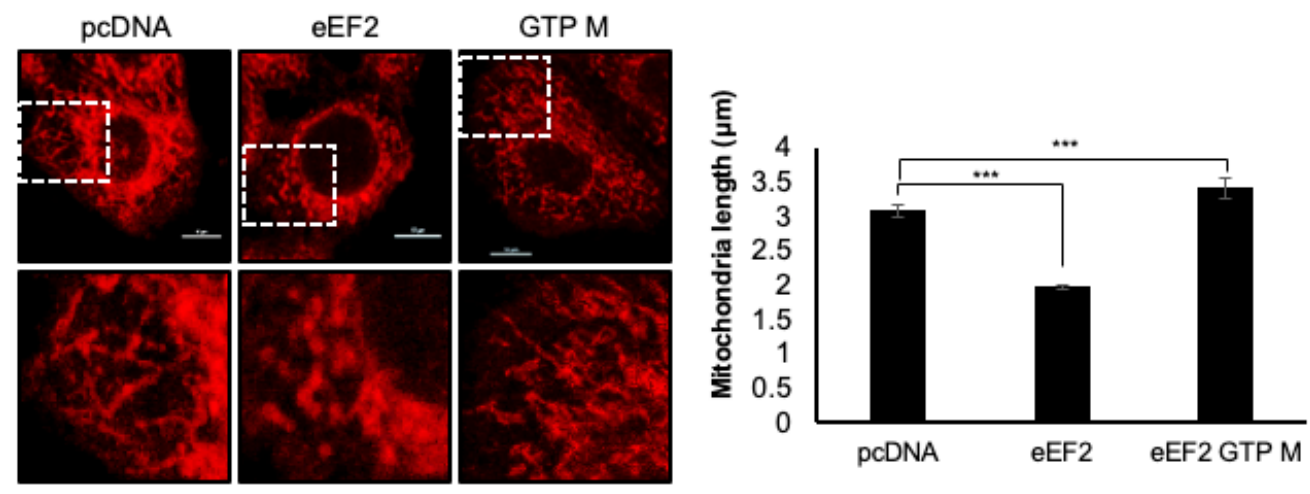

b
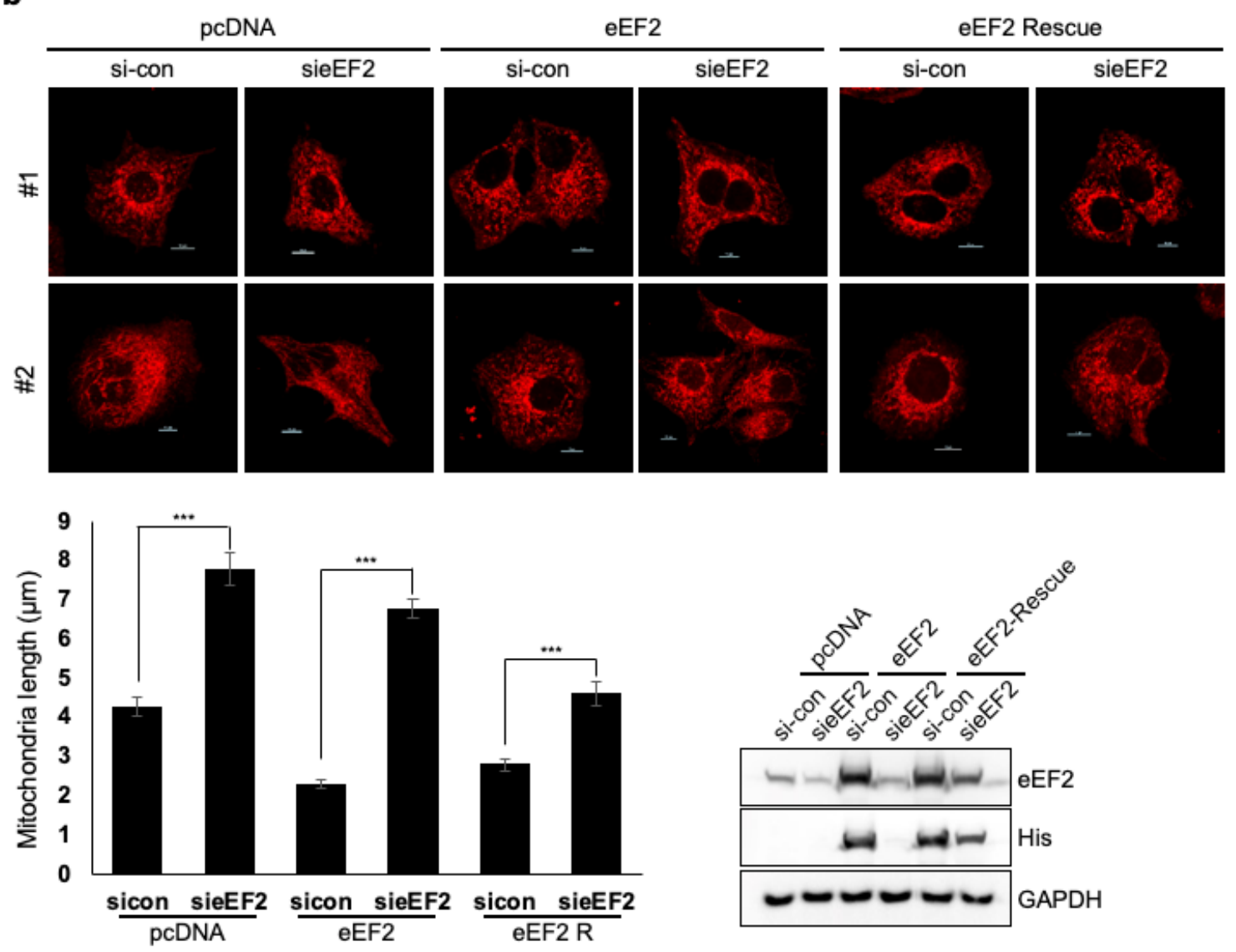

\section{Figure 5}

Validation of the effect of eEF2 on mitochondria fission. a. Effect of the eEF2 mutant on mitochondrial fission. HEK293 cells were transfected with a control vector (pcDNA 3.1 His6C), expression vectors of eEF2 or eEF2 GTP deletion mutant for 72 hours. The transfected cells were stained with Mitotracker Red $\circledast$ and observed under a confocal microscope. The lengths of mitochondria were measured using NISelements AR Analysis software (Nikon); five independent images were used $(n=5)$. b. Rescue of the eEF-2 
silencing effect on mitochondrial fission by transfection of cells with a non-degradable eEF-2 CDNA. MCF cells harboring an empty vector, a wild-type or non-degradable eEF-2 expression vector $(2 \mu \mathrm{g})$ were transfected with an eEF-2-targeted siRNA $(200 \mathrm{nM})$ for 72 hours. Mitochondrial lengths were measure as described above.

Figure 6

a

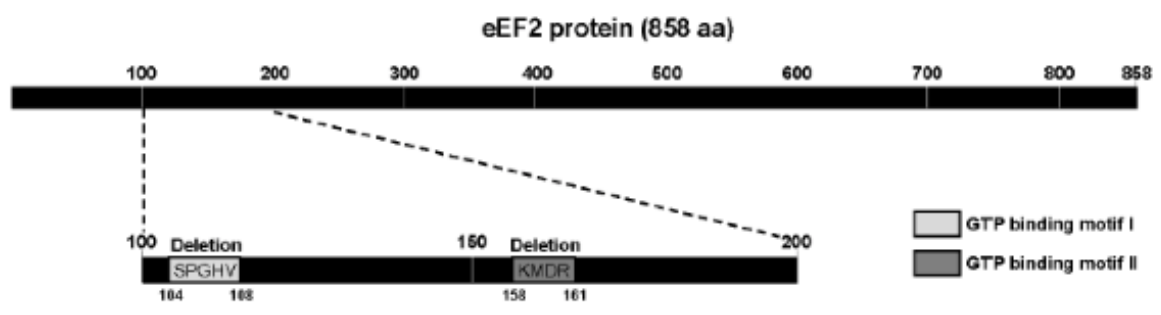

b

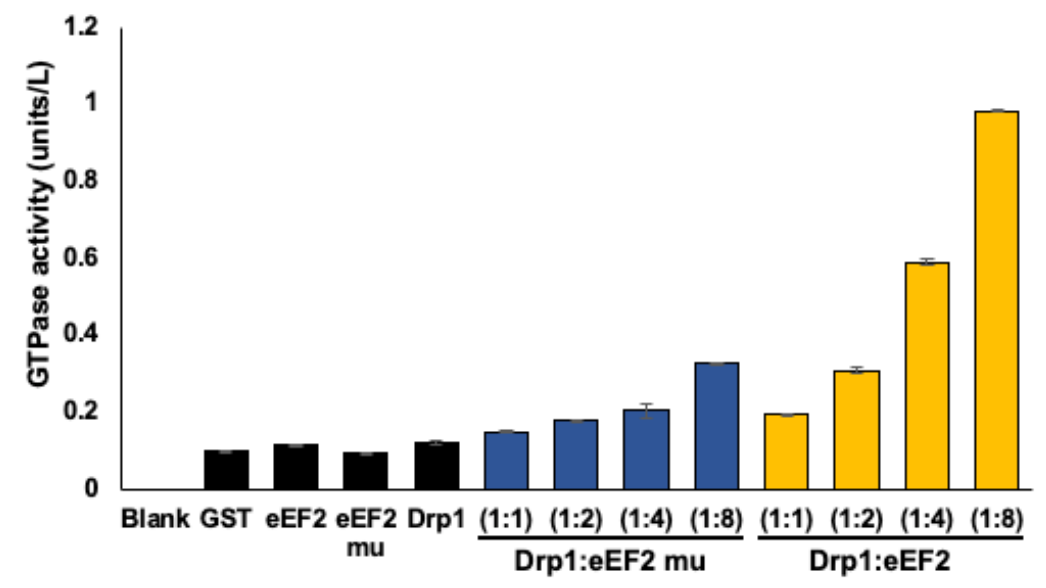

C

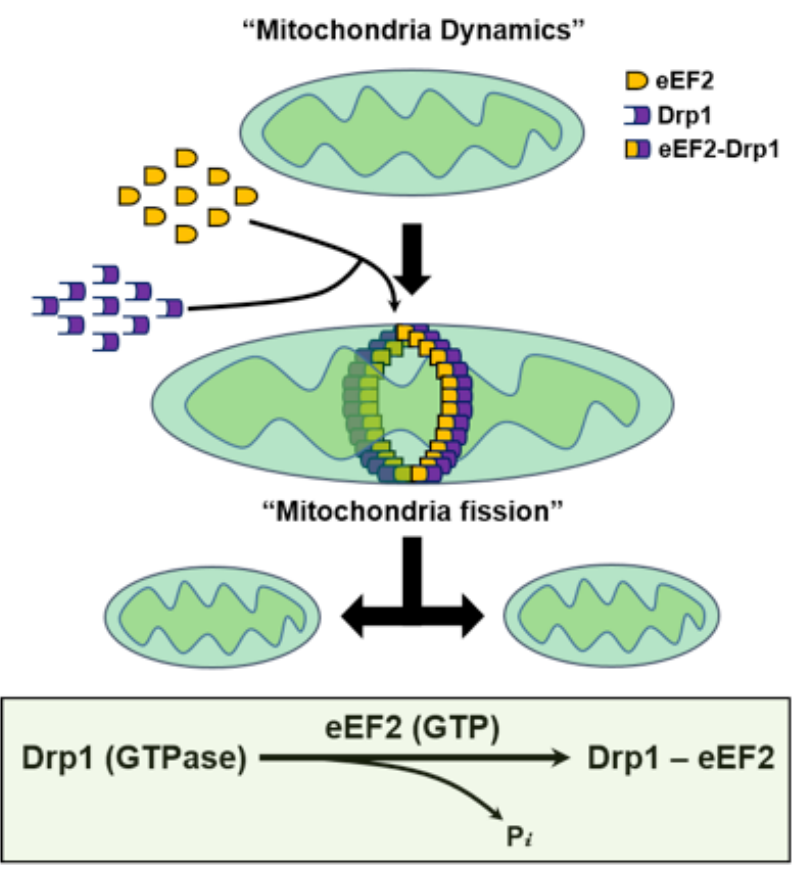

Figure 6 
Effect of eEF2 on GTPase activity of Drp1. a. Based on a functional analysis of eEF2 protein using UniPort, the GTP binding motif of eEF2 was deleted using mutagenesis PCR approach. b. eEF2, eEF2 deletion mutant (eEF2 mu) and Drp1 proteins expressed in E. Coli cells were purified using magnetic GST bead under phosphate and nucleotide free condition. Proteins of Drp1 and eEF2 or Drp1 and eEF2 GTP deletion mutant were mixed (vol:vol: $1: 1=2 \mu \mathrm{g}: 2 \mu \mathrm{g}$ ). GTPase activity was measured using the colorimetric GTPase activity assay kit from Sigma.

\section{Supplementary Files}

This is a list of supplementary files associated with this preprint. Click to download.

- SupplementalFig.1.pptx

- SupplementalFig.2movie.pptx

- Suppl.Fig.2control5.avi

- Suppl.Fig.2control4.avi

- Suppl.Fig.2eEF2siRNA.avi

- Suppl.Fig.2eEF2siRNA4.avi

- SupplementalFig.3.pptx

- SupplementalFig.4.pptx

- SupplementalFig.5.pptx 